\title{
On the Capacity of Variable Density Cellular Systems under Multicell Decoding
}

\author{
Symeon Chatzinotas, Muhammad Ali Imran, Costas Tzaras
}

\begin{abstract}
The majority of multicell-decoding cellular models preserve a fundamental assumption which has initially appeared in Wyner's model, namely the collocation of User Terminals (UTs). Although this assumption produces more tractable mathematical models, it is unrealistic w.r.t. current practical cellular systems. In this paper, we alleviate this assumption by assuming uniformly distributed UTs. The model under investigation is the uplink channel of a planar cellular array in the presence of power-law path loss and flat fading. In this context, we employ a free probability approach to evaluate the effect of UT distribution on the optimal sum-rate capacity of a variable-density cellular system.
\end{abstract}

Index Terms-Information theory, Information rates, Multiuser channels, Fading channels, Land mobile radio cellular systems, MIMO systems.

\section{INTRODUCTION}

The first concrete result for the information-theoretic capacity of the Gaussian Cellular Multiple Access Channel (GCMAC) was presented by Wyner in [1]. Using a very simple but tractable model for the cellular uplink channel, Wyner showed the importance of joint decoding at the Base Station (BS) receivers (hyper-receiver) and found the closed forms of the system capacity under the assumption of hyper-receiver (a.k.a. multicell decoding). This model triggered the interest of the research community in the cellular capacity limits and was extended in [2] to include flat fading conditions. One major assumption shared in these models was that the cell density is fixed and only physically adjacent cells interfere. Letzepis in [3], extended the model by assuming multiple-tier interference and incorporated a distance-dependent path loss factor in order to study the effect of cell density in a linear cellular array. However, the assumption of collocation of all users in a single cell was still maintained. In this paper, UTs are spatially distributed within the cell and each channel gain is affected by a distance-dependent path loss factor. The model under investigation is a GCMAC over a planar cellular array in the presence of power-law path loss and flat fading. The presented analysis is based on the principles of free probability.

\section{Channel Model And Analysis}

Assume that $K$ users per cell are uniformly distributed across a planar cellular system comprising $N$ cells. Assuming

S. Chatzinotas, M.A. Imran and C. Tzaras are with the Centre for Communication Systems Research, University of Surrey, United Kingdom, GU2 7XH e-mail: \{S.Chatzinotas, M.Imran, C.Tzaras\}@surrey.ac.uk. The work reported in this paper has formed part of the "Fundamental Limits to Wireless Network Capacity" Elective Research Programme of the Mobile VCE, www.mobilevce.com, funded by BBC, BT, Huawei, Nokia Siemens Networks, Nortel, Vodafone. flat fading, the received signal at $\mathrm{BS} n=1 \ldots N$, at time index $t$, will be given by:

$$
y^{n}[t]=\sum_{m=1}^{N} \sum_{k=1}^{K} \varsigma_{k}^{n m} g_{k}^{n m}[t] x_{k}^{m}[t]+z^{n}[t],
$$

where $x_{k}^{m}[t]$ is the $t$ th complex channel symbol transmitted by the $k$ th UT of the $m$ th cell and $\left\{g_{k}^{n m}\right\}$ are independent, strictly stationary and ergodic complex random processes in the time index $t$, which represent the flat fading processes experienced in the transmission path between the $n$th BS and the $k$ th UT in the $m$ th cell. The fading coefficients are assumed to have unit power, i.e. $\mathbb{E}\left[\left\|g_{k}^{n m}[t]\right\|^{2}\right]=1$ for all $(n, m, k)$ and all UTs are subject to an average power constraint, i.e. $\mathbb{E}\left[\left\|x_{k}^{m}[t]\right\|^{2}\right] \leq P$ for each $(m, k)$. The path loss coefficients $\varsigma_{k}^{n m}$ in the transmission path between the $m$ th BS and the $k$ th UT in the $n$th cell are calculated according to the "modified" power-law path loss model [3], [4]: $\varsigma_{k}^{n m}=\left(1+d_{k}^{n m}\right)^{-\eta / 2}$, where $d_{k}^{n m}$ denotes the distance along the line of sight of the transmission path and $\eta$ is the path loss exponent. The model can be more compactly expressed as a vector memoryless channel of the form $\mathbf{y}=\mathbf{H x}+\mathbf{z}$, where the

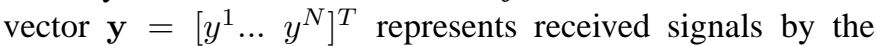
BSs, the vector $\mathbf{x}=\left[\begin{array}{lll}x_{1}^{1} & \ldots & x_{K}^{N}\end{array}\right]^{T}$ represents transmit signals by all the UTs of the cellular system and the components of vector $\mathbf{z}=\left[\begin{array}{lll}z^{1} & \ldots & z^{N}\end{array}\right]^{T}$ are independent identically distributed (i.i.d.) complex circularly symmetric (c.c.s.) random variables representing AWGN with $\mathbb{E}\left[z^{n}\right]=0, \mathbb{E}\left[\left\|z^{n}\right\|^{2}\right]=\sigma^{2}$. The channel matrix $\mathbf{H}$ can be written as $\mathbf{H}=\boldsymbol{\Sigma} \odot \mathbf{G}$, where $\boldsymbol{\Sigma}$ is a $N \times K N$ deterministic matrix, $\mathbf{G} \sim \mathcal{C N}\left(\mathbf{0}, \mathbf{I}_{N}\right)$ is a complex Gaussian $N \times K N$ matrix comprising the corresponding Rayleigh fading coefficients and $\odot$ denotes the Hadamard (element-wise) product. The entries of the $\boldsymbol{\Sigma}$ matrix are defined by the variance profile function

$$
\varsigma(u, v)=(1+d(u, v))^{-\eta / 2}
$$

where $u \in[0,1]$ and $v \in[0, K]$ are the normalized indexes for the BSs and the UTs respectively and $d(u, v)$ denotes the distance between BS $u$ and user $v$. According to [5], the asymptotic sum-rate capacity $C_{\text {opt }}$ for this model assuming a very large number of cells, is given by

$$
\begin{aligned}
C_{\mathrm{opt}} & =\lim _{N \rightarrow \infty} \frac{1}{N} \mathcal{I}(\mathbf{x} ; \mathbf{y} \mid \mathbf{H}) \\
& =\lim _{N \rightarrow \infty} \mathbb{E}\left[\frac{1}{N} \sum_{i=1}^{N} \log \left(1+\frac{\tilde{\gamma}}{K} \lambda_{i}\left(\frac{1}{N} \mathbf{H} \mathbf{H}^{\dagger}\right)\right)\right]
\end{aligned}
$$




$$
\begin{aligned}
& =\int_{0}^{\infty} \log \left(1+\frac{\tilde{\gamma}}{K} x\right) d \mathrm{~F}_{\frac{1}{N} \mathbf{H} \mathbf{H}^{\dagger}}(x) \\
& =\mathcal{V}_{\frac{1}{N} \mathbf{H} \mathbf{H}^{\dagger}}(\tilde{\gamma} / K)=K \mathcal{V}_{\frac{1}{N} \mathbf{H}^{\dagger} \mathbf{H}}(\tilde{\gamma} / K)
\end{aligned}
$$

where $\tilde{\gamma}=K N \gamma$ and $\gamma=P / \sigma^{2}$ are the system- and UTtransmit power over receiver noise ratio respectively, $\lambda_{i}(\mathbf{X})$ denotes the eigenvalues of matrix $\mathbf{X}$ and

$$
\begin{aligned}
\mathcal{V}_{\mathbf{X}}(y) & \triangleq \mathbb{E}[\log (1+y \mathbf{X})] \\
& =\int_{0}^{\infty} \log (1+y \lambda) d \mathrm{~F}_{\mathbf{X}}(\lambda)
\end{aligned}
$$

is the Shannon transform [5] of a random square Hermitian matrix $\mathbf{X}$, whose asymptotic eigenvalue distribution (a.e.d.) has a cumulative function denoted by $\mathrm{F}_{\mathbf{X}}(\lambda)$. For a complex Gaussian matrix $\mathbf{G} \sim \mathcal{C N}\left(\mathbf{0}, \mathbf{I}_{N}\right)$, the a.e.d. of $\frac{1}{N} \mathbf{G}^{\dagger} \mathbf{G}$ converges almost surely (a.s.) to the nonrandom a.e.d. of the Marčenko-Pastur law [6], whose Shannon transform is given by

$$
\mathcal{V}_{\frac{1}{N} \mathbf{G}^{\dagger} \mathbf{G}}(y) \stackrel{a . s .}{\longrightarrow} \mathcal{V}_{\mathrm{MP}}(y, K)
$$

$$
\begin{aligned}
& \text { where } \mathcal{V}_{\mathrm{MP}}(y, K)=\log \left(1+y-\frac{1}{4} \phi(y, K)\right) \\
& +\frac{1}{K} \log \left(1+y K-\frac{1}{4} \phi(y, K)\right)-\frac{1}{4 K y} \phi(y, K)
\end{aligned}
$$

$$
\begin{aligned}
& \text { and } \phi(y, K)= \\
& \left(\sqrt{y(1+\sqrt{K})^{2}+1}-\sqrt{y(1-\sqrt{K})^{2}+1}\right)^{2} .
\end{aligned}
$$

The rest of this section describes the derivation of the a.e.d. of $\frac{1}{N} \mathbf{H} \mathbf{H}^{\dagger}$ based on the analysis in [3] and using tools from the discipline of Free Probability, which was established by Voiculescu [7]. In this direction, $\frac{1}{N} \mathbf{H}^{\dagger} \mathbf{H}$ can be written as the sum of $K N \times K N$ unit rank matrices, i.e.

$$
\frac{1}{N} \mathbf{H}^{\dagger} \mathbf{H}=\sum_{i=1}^{N} \mathbf{h}_{i}^{\dagger} \mathbf{h}_{i}
$$

where $\mathbf{h}_{i} \sim \mathcal{C N}\left(\mathbf{0}, \mathbf{V}_{i}\right)$ denotes the $i$ th $1 \times K N$ row vector of $\frac{1}{\sqrt{N}} \mathbf{H}$. The covariance matrix equals $\mathbf{V}_{i}=\frac{1}{N}\left(\operatorname{diag}\left(\boldsymbol{\sigma}_{i}\right)\right)^{2}$, where $\operatorname{diag}\left(\boldsymbol{\sigma}_{i}\right)$ stands for a diagonal matrix with the elements of vector $\boldsymbol{\sigma}_{i}$ across the diagonal with $\boldsymbol{\sigma}_{i}$ being the $i$ th row of $\boldsymbol{\Sigma}$. The unit-rank matrices $\mathbf{W}_{i}=\mathbf{h}_{i}^{\dagger} \mathbf{h}_{i}$ constitute complex singular Wishart matrices with one degree of freedom and their density according to [8, Theorem 3-4] is

$$
\begin{aligned}
& f_{\mathbf{V}_{i}}\left(\mathbf{W}_{i}\right)=B_{\mathbf{V}_{i}}^{-1} \operatorname{det}\left(\mathbf{W}_{i}\right)^{1-K N} e^{-\operatorname{tr}\left(\mathbf{V}_{i}^{-1} \mathbf{W}_{i}\right)} \\
& B_{\mathbf{V}_{i}}=\pi^{K N-1} \operatorname{det}\left(\mathbf{V}_{i}\right)
\end{aligned}
$$

If $\mathbf{h}_{i}^{\dagger}=\mathbf{Q}_{i} \mathbf{S}_{i}$ is a singular value decomposition, then the density can be written as

$$
f_{\mathbf{V}_{i}}\left(\mathbf{W}_{i}\right)=B_{\mathbf{V}_{i}}^{-1} \operatorname{det}\left(\mathbf{S}_{i} \mathbf{S}_{i}^{\dagger}\right)^{1-K N} e^{-\operatorname{tr}\left(\mathbf{V}_{i}^{-1} \mathbf{Q}_{i} \mathbf{S}_{i} \mathbf{S}_{i}^{\dagger} \mathbf{Q}_{i}^{\dagger}\right)}
$$

It can be easily seen that if $\mathbf{V}_{i}=\mathbf{I}$, the matrices would be unitarily invariant [9, Definition 17.7] and therefore asymptotically free [10]. Although in our case $\mathbf{V}_{i}=\frac{1}{N} \operatorname{diag}\left(\boldsymbol{\sigma}_{i}^{2}\right)$, we assume that the asymptotic freeness still holds. Similar approximations have been already investigated in an informationtheoretic context, providing useful analytical insights and accurate numerical results [11], [12]. In this context, the Rtransform of each unit rank matrix [5, Example 2.28] is given by

$$
\mathcal{R}_{\mathbf{h}_{\mathbf{i}}{ }^{\dagger} \mathbf{h}_{\mathbf{i}}}(w)=\frac{1}{K N} \frac{\left\|\mathbf{h}_{i}\right\|^{2}}{1-w\left\|\mathbf{h}_{i}\right\|^{2}}
$$

and the asymptotic R-transform of $\mathbf{H}^{\dagger} \mathbf{H}$ is equal to the sum of the R-transforms of all the unit rank matrices [5, Theorem 2.64]

$$
\begin{aligned}
\lim _{N \rightarrow \infty} \mathcal{R}_{\frac{1}{N} \mathbf{H}^{\dagger} \mathbf{H}}(w) & \simeq \lim _{N \rightarrow \infty} \sum_{i=1}^{N} \mathcal{R}_{\mathbf{h}_{\mathbf{i}}{ }^{\dagger} \mathbf{h}_{\mathbf{i}}}(w) \\
& =\lim _{N \rightarrow \infty} \frac{1}{K N} \sum_{i=1}^{N} \frac{\left\|\mathbf{h}_{i}\right\|^{2}}{1-w\left\|\mathbf{h}_{i}\right\|^{2}} .
\end{aligned}
$$

Since the variance profile function of Equation (2) defines a rectangular block-circulant matrix with $1 \times K$ blocks which is symmetric about $u=K v$, the channel matrix $\mathbf{H}$ is asymptotically row-regular [5, Definition 2.10] and thus the asymptotic norm of $\mathbf{h}_{i}$ converges to a deterministic constant for every BS, i.e $\forall i$

$$
\lim _{N \rightarrow \infty}\left\|\mathbf{h}_{i}\right\|^{2}=\lim _{N \rightarrow \infty} \frac{1}{N} \sum_{j=1}^{K N} \varsigma_{i j}^{2}=\int_{0}^{K} \varsigma^{2}(u, v) d v,
$$

where $\varsigma_{i j}$ is the $(i, j)$ th element of the $\boldsymbol{\Sigma}$ matrix. In addition, based on the row-regularity it can be seen that

$$
\int_{0}^{K} \varsigma^{2}(u, v) d v=\int_{0}^{1} \int_{0}^{K} \varsigma^{2}(u, v) d u d v
$$

Therefore, while $\lim _{N \rightarrow \infty}$, Equation (12) can be simplified to [5, Theorem 2.31, Example 2.26]

$$
\begin{aligned}
& R_{\frac{1}{N} \mathbf{H}^{\dagger} \mathbf{H}}(w) \simeq \frac{1}{K} \int_{0}^{1} \frac{\int_{0}^{K} \varsigma^{2}(u, v) d v}{1-w \int_{0}^{K} \varsigma^{2}(u, v) d v} d u \\
&=\frac{1}{K} \frac{\int_{0}^{1} \int_{0}^{K} \varsigma^{2}(u, v) d u d v}{1-w \int_{0}^{1} \int_{0}^{K} \varsigma^{2}(u, v) d u d v} \\
&=q(\boldsymbol{\Sigma}) \frac{1}{1-K w q(\boldsymbol{\Sigma})} \\
&=R_{q(\boldsymbol{\Sigma}) \frac{1}{N} \mathbf{G}^{\dagger} \mathbf{G}(w)} \\
& \text { where } q(\boldsymbol{\Sigma}) \triangleq\|\boldsymbol{\Sigma}\|^{2} / K N^{2}
\end{aligned}
$$

with $\|\boldsymbol{\Sigma}\| \triangleq \sqrt{\operatorname{tr}\left\{\boldsymbol{\Sigma}^{\dagger} \boldsymbol{\Sigma}\right\}}$ being the Frobenius norm of the $\boldsymbol{\Sigma}$ matrix. In the asymptotic case, $q(\boldsymbol{\Sigma})$ is given by

$$
\lim _{N \rightarrow \infty} q(\boldsymbol{\Sigma})=\frac{1}{K} \int_{0}^{K} \varsigma^{2}(u, v) d v .
$$

The probability density function (p.d.f.) of the a.e.d. of $\frac{1}{N} \mathbf{H}^{\dagger} \mathbf{H}$ follows a scaled version of the Marčenko-Pastur law and hence the Shannon transform of the a.e.d. of $\frac{1}{N} \mathbf{H}^{\dagger} \mathbf{H}$ can be approximated by

$$
\mathcal{V}_{\frac{1}{N} \mathbf{H}^{\dagger} \mathbf{H}}\left(\frac{\tilde{\gamma}}{K}\right) \simeq \mathcal{V}_{\mathrm{MP}}\left(q(\boldsymbol{\Sigma}) \frac{\tilde{\gamma}}{K}, K\right)
$$




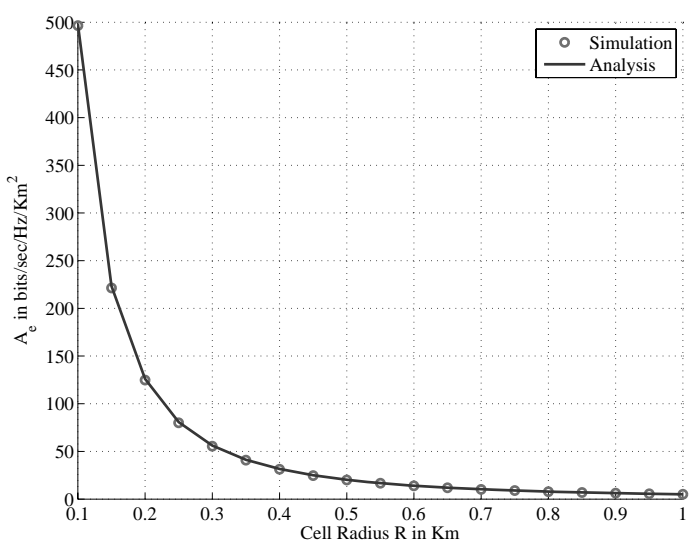

Fig. 1. Mean Area Spectral Efficiency $A_{e}$ (bits/sec/Hz/Km${ }^{2}$ ) vs. the cell radius $R$. Note that there are 127 UTs in each $\mathrm{Km}^{2}$.

It should be noted that the limiting eigenvalue distribution converges to the Marčenko-Pastur law, as long as $\boldsymbol{\Sigma}$ is asymptotically doubly-regular [5, Definition 2.10 , Theorem 2.49]. In this paper, it is shown that on the grounds of free probability, a scaled version of the Marčenko-Pastur law can be effectively utilized in cases where $\boldsymbol{\Sigma}$ is just asymptotically row-regular. Furthermore, according to [3], this analysis holds for UTs collocated with the BS in a linear cellular array. Herein, we show that the convergence holds for the case of distributed UTs. Closed-form expressions for $q(\boldsymbol{\Sigma})$ in the case of a planar cellular array are studied in [13].

\section{NumERICAL RESUlTS}

In this section, we present some simulation results in order to verify the accuracy of the asymptotic analysis for the percell sum-rate capacity $C_{\mathrm{opt}}$ of a planar cellular system with uniformly distributed UTs. In the context of the asymptotic analysis, Equations (3),(17) and (18) are utilized, while the distances $d(u, v)$ are calculated assuming that the UTs are positioned on a uniform planar grid. In the context of Monte Carlo finite-system simulations, the UT positions are randomly generated according to the considered distribution and the capacity is calculated by evaluating the capacity formula [14]:

$$
C_{\text {opt }}=(1 / N) \mathbb{E}\left[\log \operatorname{det}\left(\mathbf{I}_{N}+\gamma \mathbf{H} \mathbf{H}^{\dagger}\right)\right] .
$$

The presented results refer to the mean Area Spectral Efficiency (ASE)

$$
A_{e}=C_{\mathrm{opt}} / \pi R^{2} \quad\left(\mathrm{bits} / \mathrm{sec} / \mathrm{Hz} / \mathrm{Km}^{2}\right)
$$

averaged over a large number of fading realizations $g_{k}^{n m}$ and UT positions $d_{k}^{n m}$ and they are plotted vs. a variable cell radius $R$. In this point, it should be noted that the ASE of conventional interference-limited cellular systems has been studied in [15]. In the current setting, while scaling the cell radius, the UT density (users $/ \mathrm{Km}^{2}$ ) $K_{0}$ is kept fixed and hence the per-cell number of UTs $K=K_{0} \pi R^{2}$ and the system power scales accordingly. The path loss coefficients are calculated by considering a nominal power loss $L_{0}$ at a reference distance $d_{0}$ (see Table I for parameter values) $\varsigma(d)=\sqrt{L_{0}\left(1+d / d_{0}\right)^{-\eta}}$.
TABLE I

SIMULATION PARAMETERS

\begin{tabular}{c|c|l} 
Parameter & Symbol & Value/Range (units) \\
\hline Cell Radius & $R$ & $0.1-1 \mathrm{~km}$ \\
Reference Distance & $d_{0}$ & $1 \mathrm{~m}$ \\
Path Loss at ref. distance & $L_{0}$ & $-38 \mathrm{~dB}$ \\
Path Loss Exponent & $\eta$ & 3.5 \\
UTs per Km ${ }^{2}$ & $K_{0}$ & 127 \\
UT Transmit Power & $P_{T}$ & $200 \mathrm{~mW}$ \\
Thermal Noise Density & $N_{0}$ & $-169 \mathrm{dBm} / \mathrm{Hz}$ \\
Channel Bandwidth & $B$ & $5 \mathrm{MHz}$ \\
\hline
\end{tabular}

\section{CONCLUSION}

In conclusion, this paper has considered the capacity performance of a multicell joint decoding system under two realistic assumptions: planar coverage area and distributed UTs. Using a free probability approach, the a.e.d. of the matrix $\frac{1}{N} \mathbf{H H}^{\dagger}$ has been derived and the proposed analysis was validated with Monte Carlo simulations for variable cell-density systems. The importance of the presented methodology lies in the fact that it allows a more realistic representation of the UTs' spatial arrangement. Therefore, this approach can be employed in order to investigate various practical UT distributions and their effect on the sum-rate capacity.

\section{REFERENCES}

[1] A. Wyner, "Shannon-theoretic approach to a Gaussian cellular multipleaccess channel," IEEE Trans. Inform. Theory, vol. 40, no. 6, pp. 17131727, Nov 1994.

[2] O. Somekh and S. Shamai, "Shannon-theoretic approach to a Gaussian cellular multiple-access channel with fading," IEEE Trans. Inform. Theory, vol. 46, no. 4, pp. 1401-1425, Jul 2000.

[3] N. A. Letzepis, "Gaussian cellular multiple access channels," Ph.D. dissertation, Institute for Telecommunications Research, University of South Australia, Dec 2005.

[4] L. Ong and M. Motani, "The capacity of the single source multiple relay single destination mesh network," in IEEE International Symposium on Information Theory, Jul 2006, pp. 1673-1677.

[5] A. Tulino and S. Verdu, "Random matrix theory and wireless communications," Commun. Inf. Theory, vol. 1, no. 1, pp. 1-182, 2004.

[6] V. Marcenko and L. Pastur, "Distributions of eigenvalues of some sets of random matrices," Math. USSR-Sb., vol. 1, pp. 507-536, 1967.

[7] D. Voiculescu, "Asymptotically commuting finite rank unitary operators without commuting approximants," Acta Sci. Math., vol. 45, pp. 429431,1983

[8] T. Ratnarajah and R. Vaillancourt, "Complex random matrices and applications," Computer \& Mathematics with Applications, vol. 50, no. 3-4, pp. 399-411, Aug 2005.

[9] R. Tempo, G. Calafiore, and F. Dabbene, Randomized Algorithms for Analysis and Control of Uncertain Systems, ser. Communications and Control Engineering Series. London: Springer, 2005.

[10] F. Hiai and D. Petz, "Asymptotic freeness almost everywhere for random matrices," Acta Sci. Math. (Szeged), vol. 66, pp. 801-826, 2000.

[11] M. Peacock, I. Collings, and M. Honig, "Asymptotic spectral efficiency of multiuser multisignature CDMA in frequency-selective channels," IEEE Trans. Inform. Theory, vol. 52, no. 3, pp. 1113-1129, Mar 2006.

[12] W. Hachem, "Low complexity polynomial receivers for downlink CDMA," Signals, Systems and Computers, 2002. Conference Record of the Thirty-Sixth Asilomar Conference on, vol. 2, pp. 1919-1923 vol.2, Nov 2002.

[13] S. Chatzinotas, M. A. Imran, C. Tzaras., "Optimal information theoretic capacity of the planar cellular uplink channel," to appear in Proc. IEEE SPAWC 2008, Jul 2008.

[14] I. E. Telatar, "Capacity of multi-antenna Gaussian channels," European Transactions on Telecommunications, vol. 10, no. 6, pp. 585-595, Nov 1999.

[15] M. Alouini and A. Goldsmith, "Area spectral efficiency of cellular mobile radio systems," IEEE Trans. Veh. Technol., vol. 48, no. 4, pp. 1047-1066, Jul 1999. 